\title{
Does Weight Impact Anidulafungin Pharmacokinetics?
}

\author{
Vincent J. Lempers ${ }^{1,2}$ - Anne van Rongen ${ }^{3,4}$ - Eric P. van Dongen ${ }^{5}$. \\ Bert van Ramshorst $^{6}$ - David M. Burger ${ }^{1,2}$ - Rob E. Aarnoutse ${ }^{1,2}$. \\ Catherijne A. Knibbe ${ }^{3,4} \cdot$ Roger J. Brüggemann ${ }^{1,2}$
}

Published online: 3 May 2016

(C) The Author(s) 2016. This article is published with open access at Springerlink.com

\begin{abstract}
Bodyweight has been shown to influence anidulafungin exposure, but data from obese patients are lacking. We determined anidulafungin pharmacokinetics (100-mg single dose) in eight morbidly obese subjects (body mass index $>40 \mathrm{~kg} / \mathrm{m}^{2}$ ). Anidulafungin exposure was on average $32.5 \%$ lower compared with the general patient population, suggesting dose increases may be required in this population.
\end{abstract}

Electronic supplementary material The online version of this article (doi:10.1007/s40262-016-0401-8) contains supplementary material, which is available to authorized users.

Roger J. Brüggemann

roger.bruggemann@radboudumc.nl

1 Department of Pharmacy, Radboud University Medical Center, 864, PO Box 9101 HB, Nijmegen 6500, The Netherlands

2 Radboud Institute for Health Sciences, Nijmegen, The Netherlands

3 Department of Clinical Pharmacy, St. Antonius Hospital, Nieuwegein, The Netherlands

4 Division of Pharmacology, Leiden Academic Centre for Drug Research, Leiden University, Leiden, The Netherlands

5 Department of Anaesthesiology, Intensive Care and Pain Management, St. Antonius Hospital, Nieuwegein, The Netherlands

6 Department of Surgery, St. Antonius Hospital, Nieuwegein, The Netherlands

\section{Key Points}

Anidulafungin exposure was on average $32.5 \%$ lower compared with the general patient population.

To normalize the exposure to population values, increasing the anidulafungin maintenance dose by $50 \%$ (i.e., $150 \mathrm{mg}$ ) could be considered.

To achieve adequate exposure at the beginning of therapy, increasing the loading dose by $50 \%$ (i.e., $300 \mathrm{mg}$ ) could be considered.

\section{Introduction}

The global prevalence of overweight and obesity has increased at an alarming rate during the previous few decades. If recent trends continue, nearly $58 \%$ of the world's adult population will be overweight or obese in 2030 [1]. Obesity increases the risk of a wide array of comorbidities and is an established risk factor for nosocomial infections. Because obese patients are subject to a variety of (patho)physiological changes compared with non-obese patients, the pharmacokinetic (PK) and/or pharmacodynamic profile of antimicrobials might be altered [2].

Anidulafungin is an echinocandin antifungal agent approved for the intravenous treatment of invasive candidiasis and candidemia [3]. At standard doses (200-mg loading dose, 100-mg maintenance dose), anidulafungin displays linear PK, with a volume of distribution close to total body water $(0.6 \mathrm{~L} / \mathrm{kg})$, a clearance of about $1 \mathrm{~L} / \mathrm{h}$, and

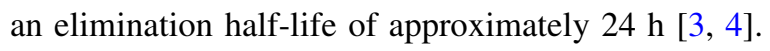


The PK of anidulafungin has been well described in healthy subjects and several patient populations [4-10], although the weight range in these studies was small. Weight has been reported to be an influential factor on anidulafungin exposure [4, 8-10]. This may therefore result in suboptimal exposure of anidulafungin in obese patients, possibly requiring a different dosing strategy in this population. In this study, we aim to describe the PK of anidulafungin in morbidly obese subjects with body mass index $(\mathrm{BMI})>40 \mathrm{~kg} / \mathrm{m}^{2}$.

\section{Materials and Methods}

\subsection{Study Design and Subjects}

This open-label phase IV study (ClinicalTrials.gov Identifier: NCT02021123) was approved by the Ethics Committee of the Radboud University Medical Center in 2014 and conducted in accordance with the declaration of Helsinki. Before inclusion, all subjects gave written informed consent.

From August until October 2014, adult morbidly obese subjects (BMI $>40 \mathrm{~kg} / \mathrm{m}^{2}$; with no fungal infection) undergoing laparoscopic gastric bypass or sleeve surgery were eligible for inclusion. Subjects were excluded in the case of hypersensitivity to echinocandins and/or abuse of alcohol or drugs for the previous 3 months.

\subsection{Study Procedure}

Upon inclusion, patient demographics, clinical characteristics, and concomitant medications were reported. Subjects received a single intravenous 100-mg dose of anidulafungin before laparoscopic bariatric surgery. The anidulafungin infusion was administered $2.5 \mathrm{~h}$ before induction of anesthesia with an infusion rate of $1.1 \mathrm{mg} / \mathrm{min}$ [3]. There were no restrictions in the protocol with regard to concomitant medication.

A PK curve was drawn at predefined times of $T=0.5$, $1,1.5$ (end of infusion), 2, 4, 6, 8, 10, 12, 24, and 48 h postinfusion $(n=11)$. Blood samples were collected in lithium-heparin-containing tubes (non-gel) and centrifuged at $1900 \times g(3000 \mathrm{rpm})$ for $5 \mathrm{~min}$ at $4{ }^{\circ} \mathrm{C}$ within $30 \mathrm{~min}$ of collection. Plasma was immediately stored at $-80{ }^{\circ} \mathrm{C}$. Anidulafungin samples were measured by ultra-performance liquid with fluorescence detection [8].

\subsection{Analytical Assay}

Anidulafungin samples were measured by ultra-performance liquid chromatography with fluorescence detection (dynamic range for anidulafungin in plasma:
0.008-8.43 $\mathrm{mg} / \mathrm{L}$ with a concentration-dependent accuracy range $[n=15]$ of $94.2-103.5 \%$ ). A seven-point calibration curve with three quality-control samples was used. Intraday precision ranged between 0.87 and $1.84 \%(n=5)$ and interday precision varied between 0.53 and $1.58 \%(n=15)$. Anidulafungin recovery was $93 \%$. Stability of anidulafungin remained unchanged by three freeze-thaw cycles.

\subsection{Safety}

Blood samples for the purpose of laboratory safety were collected at $T=0,24$, and $48 \mathrm{~h}$ for the determination of biochemical and hematological parameters (sodium, potassium, chloride, calcium, phosphate albumin, blood urea nitrogen, aspartate aminotransaminase, alanine aminotransferase, gamma-glutamyltransferase, alkaline phosphatase, bilirubin (total), lactate dehydrogenase, C-reactive protein, triglycerides, creatinine kinase, creatinine, uric acid, hemoglobin, hematocrit, white blood cells differential, platelets, and red blood cell count).

Adverse events (AEs) were reported regardless of potential relationship to anidulafungin (including adverse drug reactions, illness that developed during the study, exacerbations of pre-existing illness, or abnormal laboratory values requiring intervention or diagnostic evaluation) until discharge.

There were no restrictions in the protocol with regard to concomitant medication.

\subsection{Anidulafungin PK Data Analysis}

PK parameters were calculated using non-compartmental analysis (Phoenix WinNonlin 6.3; Pharsight Corp, Mountain View, CA, USA). The area under the plasma concentration-time curve from 0 to time of last sample $\left(\mathrm{AUC}_{0-48}\right)$ was calculated using the linear up-log down trapezoidal rule. The AUC from 0 to infinity $\left(\mathrm{AUC}_{0-\mathrm{inf}}\right)$ was determined as follows:

$\mathrm{AUC}_{0-\text { inf }}=\mathrm{AUC}_{0-48}+$ last observed concentration $/ k_{\mathrm{e}}$.

Anidulafungin exposure in this cohort of morbidly obese subjects was compared with the exposure in the general patient population [10], given that $\mathrm{AUC}_{0-\text { inf }}$ (single dose $)=\mathrm{AUC}_{0-24}($ steady state $)$.

Maximum plasma concentration was directly observed from the data. Total body clearance was calculated as $D /$ $\mathrm{AUC}_{0-\text { inf }}$ and volume of distribution $\left(V_{\mathrm{D}}\right)$ was calculated as $D / \mathrm{AUC}_{0-\text { inf }} \times k_{\mathrm{e}}$. Half-life was calculated by $\ln (2) / k_{\mathrm{e}}$. Elimination rate constant $\left(k_{\mathrm{e}}\right)$ was estimated by log-linear regression of the terminal portions (minimum of four points, user defined) of the plasma concentration-vs.-time curves. 


\subsection{Statistical Analysis}

Descriptive statistics (e.g., geometric mean, range, interpatient variability [calculated as geometric coefficient of variation]) were calculated for anidulafungin PK parameters. A Spearman's correlation was run to assess the correlation between AUC and weight in SPSS 20.0 (SPSS Inc., Chicago, IL, USA). A $p$ value of $<0.05$ was considered statistically significant. Using a power calculation (alpha $=0.05$, power $=0.8$ ) based on the exposure and standard deviation in the general patient population $[9,10]$ and the assumption of a mean $\mathrm{AUC}_{0-\text { inf }}$ of $75 \mathrm{mg} \times \mathrm{h} / \mathrm{L}$ in the target obese population, this would require eight patients.

\section{Results}

\subsection{Subjects}

Eight subjects (three male, five female; all Caucasian) were included. Median (range) age was 43 years (29-66 years). Geometric mean (range) weight, BMI, lean body mass (calculated according to Janmahasatian et al. [11], and body surface area and waist/hip ratio were $144.7 \mathrm{~kg}$ (124.1-166.5), $\quad 48.9 \mathrm{~kg} / \mathrm{m}^{2} \quad(39.9-57.6), \quad 72.4 \mathrm{~kg}$ (58.3-91.0), $2.49 \mathrm{~m}^{2}$ (2.20-2.78), and 0.93 (0.85-1.11), respectively (Table 1). Individual and average plasma concentration-time curves of anidulafungin are shown in Fig. 1.

\subsection{Anidulafungin PK}

Geometric mean (range) $\mathrm{PK}$ parameters were: $\mathrm{AUC}_{0-\text { inf }}$ $72.9 \mathrm{mg} \times \mathrm{h} / \mathrm{L} \quad(46.3-100.1), \quad \mathrm{AUC}_{0-48} \quad 54.1 \mathrm{mg} \times \mathrm{h} / \mathrm{L}$ (35.0-68.9), maximum plasma concentration $3.2 \mathrm{mg} / \mathrm{L}$ (2.6-4.1), volume of distribution 46.9 L (39.6-56.7), clearance $1.4 \mathrm{~L} / \mathrm{h}$ (1.0-2.2), half-life $23.7 \mathrm{~h}$ (17.0-29.8), and $k_{\mathrm{e}} 0.0291 / \mathrm{h}(0.023-0.041)$, see Table 1 . In total, $35.1 \%$ of the $\mathrm{AUC}_{0-48}$ was extrapolated to $\mathrm{AUC}_{0 \text {-inf. }}$. Limited inter-individual variability was seen with $\mathrm{AUC}_{0-\text { inf }}$ (coefficient of variation (CV) of $20.7 \%$; calculated on the arithmetic mean). No concurrent medications known to significantly influence anidulafungin PK were administered [3].

A strong non-significant negative correlation between anidulafungin $\mathrm{AUC}_{0-\mathrm{inf}}$ and absolute bodyweight or anidulafungin $\mathrm{AUC}_{0-\mathrm{inf}}$ and body surface area was found: $r_{\mathrm{s}}(8)=-0.6429, \quad p=0.096 \quad$ and $\quad r_{\mathrm{s}}(8)=-0.7066$, $p=0.058$, respectively. Other parameters such as lean body mass resulted in poorer correlations. For the purpose of comparison with other studies, we also calculated the arithmetic mean AUC. The mean $\mathrm{AUC}_{0-\text { inf }}(74.4 \mathrm{mg} \times \mathrm{h} /$
L, CV $20.7 \%$ ); range 46.3-100.1) following a single dose in our patient population was on average $32.5 \%$ lower compared with the mean $\mathrm{AUC}_{0-24}$ at steady state in the general patient population $(110.3 \mathrm{mg} \times \mathrm{h} / \mathrm{L}, \mathrm{CV} 32.5 \%)$ [10]. With this, the $\mathrm{AUC}_{0-\text { inf }}$ in this cohort of morbidly obese patients is at the lower end of the exposure distribution of the general patient population.

\subsection{Safety}

No serious AEs were reported. All single-dose infusions were well tolerated. Subjects experienced 48 new or aggravated AEs during follow-up (laboratory safety until $48 \mathrm{~h}$ and clinical AEs until discharge), of which 16 (33.3\%) were possibly related to anidulafungin. These AEs are most likely related to the surgical procedure (e.g., increase in aspartate aminotransferase, alanine aminotransferase, creatinine kinase, nausea, headache) but a relation with anidulafungin infusion could not be excluded. All AEs were mild, transient, and resolved spontaneously.

\section{Discussion}

To our knowledge, this is the first clinical study investigating the influence of extreme bodyweight (BMI $>40 \mathrm{~kg} /$ $\mathrm{m}^{2}$ ) on anidulafungin PK. The findings from this study show that this cohort of morbidly obese subjects has a lower exposure compared with the exposure in non-obese individuals as described in the literature $[5,10]$. The interindividual variability of anidulafungin in this population is comparable to healthy non-obese volunteers [7].

Previously, it has been suggested that increased body size results in lower anidulafungin exposure, as weight was identified as a covariate affecting anidulafungin clearance $[4,9,10]$ and central volume of distribution $[4,8]$. Although the weight range was limited in those studies (only seven patients were $>120 \mathrm{~kg}$ ), it was predicted that anidulafungin exposure could be $30 \%$ lower in a typical $150-\mathrm{kg}$ male patient compared with a typical $60-\mathrm{kg}$ male patient [10]. In the study of Liu et al., a patient weighing $240 \mathrm{~kg}$ was included, in whom a dose increase to $150 \mathrm{mg} /$ day resulted in exposure comparable to other critically ill patients, albeit at the lower end of the AUC range $\left(\mathrm{AUC}_{0-24} 92.7\right.$ vs. $55.3 \mathrm{mg} \times \mathrm{h} / \mathrm{L} ; 37 \mathrm{mg} \times \mathrm{h} / \mathrm{L}$ if extrapolated to $100 \mathrm{mg}$ ) [7]. The above findings of decreasing exposure as a function of weight were also observed with the other echinocandins, caspofungin and micafungin $[12,13]$.

We show that none of the morbidly obese patients included in our study obtain the AUC of the general patient population at standard doses of anidulafungin (Table 1), thereby possibly introducing the risk of therapeutic failure. 


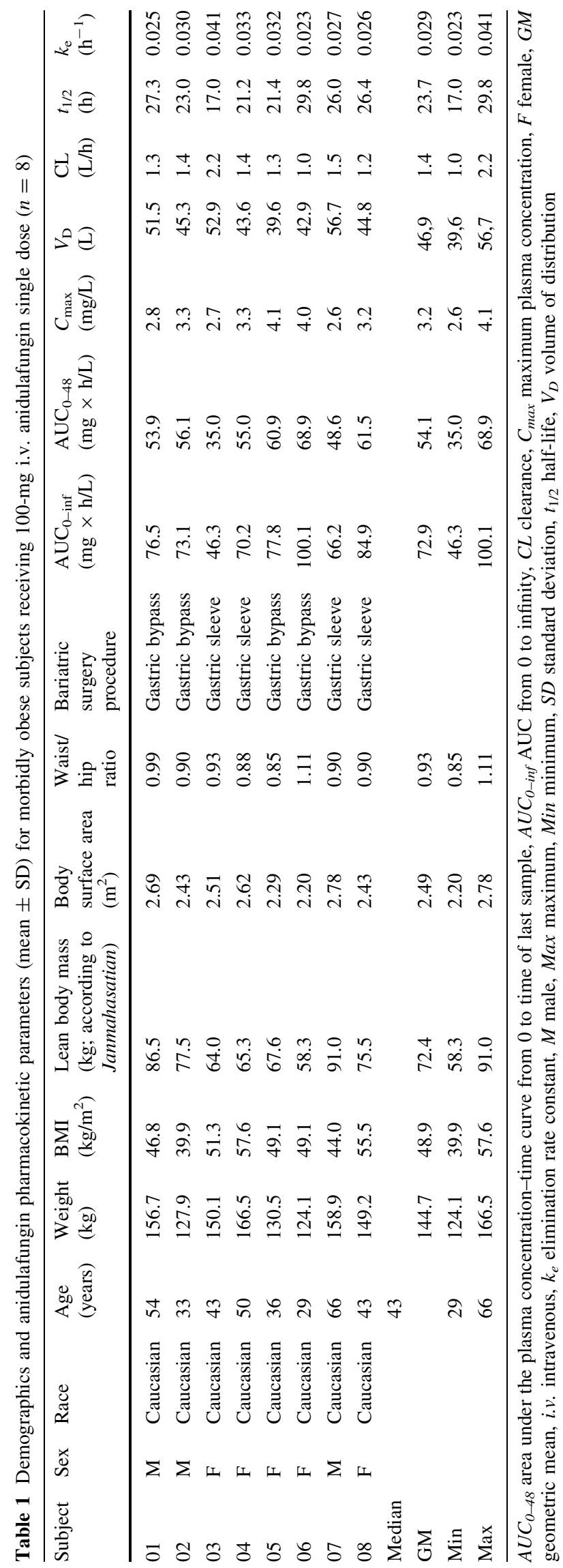




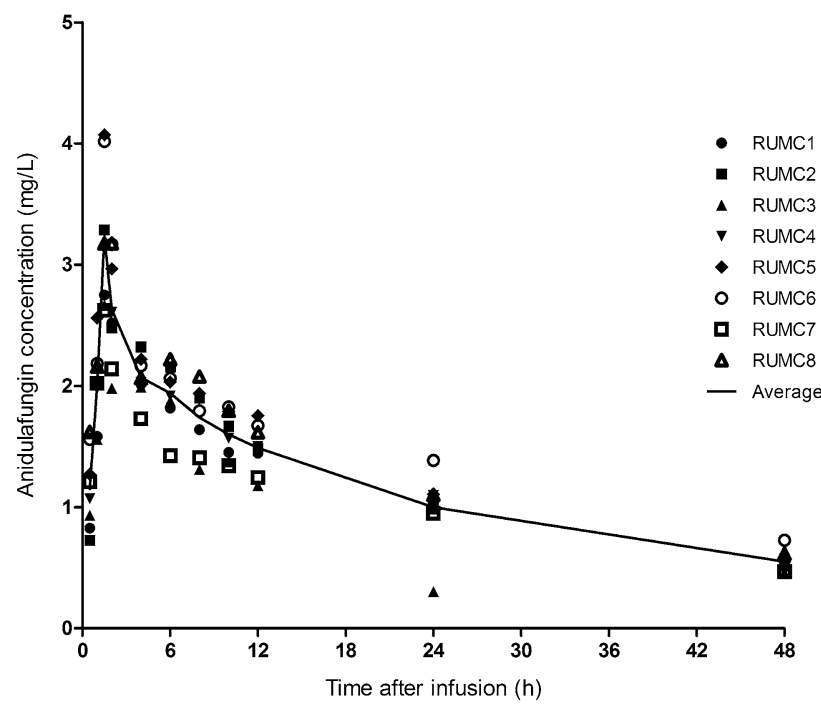

Fig. 1 Individual and average plasma concentration-time curves of anidulafungin $(n=8)$

No specific clinical target AUC value for anidulafungin is established. In addition, it must be noted that a successful clinical response was observed in patients with invasive candidiasis (including candidemia) and low exposure in another study [10]. Our hypothesis is that a favorable response is likely associated with an infection with very susceptible species. Nevertheless, morbidly obese patients infected with pathogens with reduced susceptibility are still at risk for therapeutic failure. Considering the fact that anidulafungin is well tolerated in doses up to $300 \mathrm{mg}$ [8], and pathogen susceptibility at the start of therapy is often unknown, we propose an approach to adapt the empiric dose to achieve the general population average exposure $\left(\mathrm{AUC}_{0-24} 110 \mathrm{mg} \times \mathrm{h} / \mathrm{L}\right)$ in this cohort of patients $\left(\mathrm{BMI}>40 \mathrm{~kg} / \mathrm{m}^{2}\right)$ [10]. To normalize the exposure to population values, increasing the anidulafungin maintenance dose to $150 \mathrm{mg}(+50 \%)$ would proportionally increase the $\mathrm{AUC}_{0-24}$ to nearly $110 \mathrm{mg} \times \mathrm{h} / \mathrm{L}$ (based on linear kinetics) [3]. In parallel, increasing the loading dose by $50 \%$ (i.e., $300 \mathrm{mg}$ ) could be considered to achieve adequate exposure at the beginning of therapy $[5,10]$.

A different approach would be to increase the dose based on clinical failure or by using therapeutic drug monitoring (individualized drug dosing based on the measurement and interpretation of drug concentrations taking into account pathogen susceptibility). The first option will likely save drug costs. The latter approach is a more personalized schedule that can be deployed in patients with a high a priori risk of low exposure such as the intensive care unit population [10]. Being obese may have an additive effect on exposure. In other words, the exposure in critically ill, morbidly obese patients with candidemia/invasive candidiasis may be even more pronounced compared with an intensive care unit population or obesity alone. The use of therapeutic drug monitoring in such a clinical situation deserves further study.

Our study is conducted with a relatively small sample size, without a non-obese control group. Ideally, anidulafungin PK in this cohort of morbidly obese patients is directly compared with PK in non-obese individuals also receiving a single $100-\mathrm{mg}$ dose. We did not have a control group in our study design as this study was established for exploratory purposes. Instead, we calculated $\mathrm{AUC}_{0-\text { inf }}$ after a single dose of $100 \mathrm{mg}$ anidulafungin, which would allow for comparisons with $\mathrm{AUC}_{0-24}$ at steady state in the literature (next to a comparison with single-dose studies). Because of the relatively long half-life of anidulafungin of $23.7 \mathrm{~h}$ (Table 1), more than $20 \%$ of the $\mathrm{AUC}_{0-48}$ was extrapolated to $\mathrm{AUC}_{0-\mathrm{inf}}(35.1 \%)$, which may bias this estimation of $\mathrm{AUC}_{0-\text { inf. }}$ We expect this bias to be minimal. Ideally, sampling up until $72 \mathrm{~h}$ would have been done to better estimate $\mathrm{AUC}_{0-\text { inf }}$. In our situation, patients were discharged after $48 \mathrm{~h}$, so this was the compromise between patient burden and PK results. A strong negative but nonsignificant correlation between body weight and AUC as well as body surface area and AUC was found. We want to highlight that these results should be interpreted with caution as there is only eight people in the analysis and the relation is not significant. Therefore, these results should be regarded as explorative for anidulafungin PK in obese subjects.

\section{Conclusion}

The lower anidulafungin exposure in our 'healthy' morbidly obese subjects compared with literature values in non-obese patients suggests that anidulafungin dosing could be optimized in (extreme) morbidly obese patients with fungemia. As a priori dosing information regarding the appropriate dose of anidulafungin for heavy patients is lacking, the results of the current study show that increases of both the loading dose and maintenance dose should be considered in patients with a BMI $>40 \mathrm{~kg} / \mathrm{m}^{2}$. We propose a $50 \%$ increased loading and maintenance dose for morbidly obese patients.

Acknowledgments We express gratitude to all patients who participated in this study. We thank Angela Colbers for her suggestions and comments as well as Brigitte Bliemer (bariatric nurse), and the medical, nursing, and analytical staff.

\section{Compliance with Ethical Standards}

Funding This work was supported by Pfizer, Inc. (investigatorsponsored research study). 
Disclosures Roger Brüggemann declares that he has served as a consultant to and has received unrestricted and research grants from Astellas Pharma Inc., Gilead Sciences, Merck Sharpe and Dohme Corp., and Pfizer Inc. All payments were invoiced by the Radboud University Medical Center. Vincent Lempers, Anne van Rongen, Eric van Dongen, Bert van Ramshorst, David Burger, Rob Aarnoutse, and Catherijne Knibbe declare that they have no conflicts of interest.

Open Access This article is distributed under the terms of the Creative Commons Attribution-NonCommercial 4.0 International License (http://creativecommons.org/licenses/by-nc/4.0/), which permits any noncommercial use, distribution, and reproduction in any medium, provided you give appropriate credit to the original author(s) and the source, provide a link to the Creative Commons license, and indicate if changes were made.

\section{References}

1. Kelly T, Yang W, Chen CS, Reynolds K, He J. Global burden of obesity in 2005 and projections to 2030. Int J Obes (Lond). 2008;32(9):1431-7.

2. Knibbe CA, Brill MJ, van Rongen A, Diepstraten J, van der Graaf PH, Danhof M. Drug disposition in obesity: toward evidencebased dosing. Annu Rev Pharmacol Toxicol. 2015;6(55):149-67.

3. European Medicines Agency (EMA). Ecalta; summary of product characteristics (last updated: 04/08/2015). http://www.ema. europa.eu/docs/en_GB/document_library/EPAR_-_Product_ Information/human/000788/WC500020673.pdf. Accessed 3 Apr 2016.

4. Dowell JA, Knebel W, Ludden T, Stogniew M, Krause D, Henkel T. Population pharmacokinetic analysis of anidulafungin, an echinocandin antifungal. J Clin Pharmacol. 2004;44(6):590-8.
5. Dowell JA, Stogniew M, Krause D, Henkel T, Damle B. Lack of pharmacokinetic interaction between anidulafungin and tacrolimus. J Clin Pharmacol. 2007;47(3):305-14.

6. Dowell JA, Stogniew M, Krause D, Damle B. Anidulafungin does not require dosage adjustment in subjects with varying degrees of hepatic or renal impairment. J Clin Pharmacol. 2007;47(4):461-70.

7. Liu P, Ruhnke M, Meersseman W, Paiva JA, Kantecki M, Damle B. Pharmacokinetics of anidulafungin in critically ill patients with candidemia/invasive candidiasis. Antimicrob Agents Chemother. 2013;57(4):1672-6.

8. Brüggemann RJ, Van Der Velden WJ, Knibbe CA, Colbers A, Hol S, Burger DM, et al. A rationale for reduced-frequency dosing of anidulafungin for antifungal prophylaxis in immunocompromised patients. J Antimicrob Chemother. 2015;70(4): 1166-74.

9. Liu P, Mould DR. Population pharmacokinetic analysis of voriconazole and anidulafungin in adult patients with invasive aspergillosis. Antimicrob Agents Chemother. 2014;58(8):471826.

10. Liu P. Population pharmacokinetic-pharmacodynamic analysis of anidulafungin in adult patients with fungal infections. Antimicrob Agents Chemother. 2013;57(1):466-74.

11. Janmahasatian S, Duffull SB, Ash S, Ward LC, Byrne NM, Green B. Quantification of lean bodyweight. Clin Pharmacokinet. 2005;44(10):1051-65.

12. Hall RG, Swancutt MA, Gumbo T. Fractal geometry and the pharmacometrics of micafungin in overweight, obese, and extremely obese people. Antimicrob Agents Chemother. 2011; 55(11):5107-12.

13. Hall RG 2nd, Swancutt MA, Meek C, Leff R, Gumbo T. Weight drives caspofungin pharmacokinetic variability in overweight and obese people: fractal power signatures beyond two-thirds or threefourths. Antimicrob Agents Chemother. 2013;57(5):2259-64. 